\title{
Hyperdiploidy associated with T315I mutation in BCR-ABL kinase domain in an accelerated phase-chronic myeloid leukemia case
}

\author{
Walid Al-Achkar ${ }^{1 *}$, Faten Moassass ${ }^{1}$, Adnan Ikhtiar², Thomas Liehr ${ }^{3}$, Moneeb Abdullah Kassem Othman ${ }^{3}$
} and Abdulsamad Wafa ${ }^{1}$

\begin{abstract}
Background: Chronic myeloid leukemia $(C M L)$ is genetically characterized by the occurrence of a reciprocal translocation $\mathrm{t}(9 ; 22)(\mathrm{q} 34 ; \mathrm{q} 11)$, resulting in a $\mathrm{BCR} / \mathrm{ABL}$ gene fusion on the derivative chromosome 22 , i.e. the Philadelphia (Ph) chromosome. During CML progression 60-80\% of the cases acquire additional genetic changes. Even though hyperdiploidy is not a rare finding in advanced phase-CML, hyperdiploidy together with a T315I kinase domain (KD) mutation in the BCR-ABL gene has not yet been reported.

Results: A complete cytogenetic and molecular cytogenetic analysis; molecular biology methods such as quantitative reverse transcription polymerase chain reaction (RQ-PCR) and allele-specific oligonucleotide (ASO)-PCR; and immunophenotypically confirmed CML in acceleration phase (AP). Our case revealed the presence of hyperdiploidy including multiple copies of the Ph chromosome, presence of b3a2 fusion transcript,T315I mutation in BCR-ABL KD in pre imatinib mesylate (IM) treatment. The ratio of BCR-ABL/ABL expression in post nilotinib treatment was $0.07 \%$ on international scale.

Conclusions: The patient demonstrated a good response to nilotinib after imatinib failure; while the hyperdiploid clone disappeared the T315I mutation remained during follow-up. The underlying mechanisms and prognostic implications of these cytogenetic abnormalities are discussed.
\end{abstract}

Keywords: Chronic myeloid leukemia, Philadelphia chromosome, Hyperdiploidy, T315I mutation, Prognostic factors

\section{Background}

Chronic myeloid leukemia (CML) is a disorder characterized by the formation of granulocytes in connection with a translocation involving chromosomes 9 and 22 . Resulting derivative chromosome 22 is called Philadelphia chromosome $(\mathrm{Ph})[1]$. A simple reciprocal translocation of $\mathrm{t}(9 ; 22)(\mathrm{q} 34 ; \mathrm{q} 11)$ can be observed as single aberration in the early stage chronic phase $(\mathrm{CP})$ whereas in most advanced cases this change is accompanied by other chromosomal aberrations. In fact, a chimeric gene BCR-ABL gene and protein is formed. The fused gene has an oncogenic activity and encodes for an active tyrosine kinase [2]. Hyperdiploidy is not common in CML

\footnotetext{
*Correspondence: ascientific@aec.org.sy

${ }^{1}$ Human Genetics Division, Department of Molecular Biology and

Biotechnology, Atomic Energy Commission, P.O. Box 6091, Damascus, Syria

Full list of author information is available at the end of the article
}

cases [3] and it is a common finding in advanced phaseCML patients $[4,5]$ and it was already in one CML-CP patient as a secondary chromosomal aberration after imatinib mesylate (IM) therapy [6].

Imatinib (IM = Glivec, formerly STI571) is a chemically designed drug which blocks BCR/ABL1 tyrosine kinase activity and is successfully used in CML patients [7]. Nilotinib is a second generation tyrosine kinase inhibiter (TKI) with improved target specificity and potency. Nilotinib acts like IM and binds to the kinase domain (KD) of $A B L 1$. This prevents the formation of an active conformation, catalyzing the transduction of the BCR-ABL signal [8]. Nilotinib was approved for CML treatment in $\mathrm{CP}$ and AP especially in cases showing IM resistance due to mutations in BCR-ABL tyrosine kinase geneor intolerance to prior successful IM therapy $[9,10]$. T315I is one of

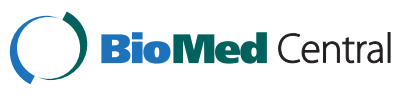

(c) 2014 Al-Achkar et al.; licensee BioMed Central. This is an Open Access article distributed under the terms of the Creative Commons Attribution License (http://creativecommons.org/licenses/by/4.0), which permits unrestricted use, distribution, and reproduction in any medium, provided the original work is properly credited. The Creative Commons Public Domain Dedication waiver (http://creativecommons.org/publicdomain/zero/1.0/) applies to the data made available in this article, unless otherwise stated. 
the most frequent mutations in the BCR-ABL domain and has been associated with TKI resistance of 1st and 2nd generation drugs [11].

Here we presented a new CML case in AP pre IM treatment with hyperdiploidy including more than one copy of the $\mathrm{Ph}$ chromosome, presence of b3a2 fusion transcript, a T315I mutation in the BCR-ABL KD, and a ratio of $\mathrm{BCR}-\mathrm{ABL} / \mathrm{ABL} 1$ expression post nilotinib treatment was $0.07 \%$ on international scale (IS). This patient demonstrated a good response to nilotinib after imatinib failure.

\section{Case presentation}

In June 2012 a 53-year-old male was diagnosed as suffering from CML. Physical examination revealed hepatosplenomegaly, several skin nodules $(2 \mathrm{~cm})$ in different locations such as neck and armpit (data not shown), anemia, thrombocytopenia, fever, fatigue, and loss of weight were the indicative symptoms. The patient's hematologic parameters were white blood cells (WBC) of $52.2 \times 10^{9} / 1$ (15\% of cells were blasts), red blood cell (RBC) count was $2.50 \times 10^{6} / \mathrm{mm}^{3}$, hemoglobin level was $7.1 \mathrm{~g} / \mathrm{dl}$ and the platelet count was $90 \times 10^{9} / 1$. Serum lactate dehydrogenase value $(\mathrm{LDH})$ was $1,851 \mathrm{U} / \mathrm{l}$ (normal level $<460 \mathrm{U} / \mathrm{l})$. The patient was diagnosed as CML-AP according to WHO recommendations and in an intermediate Sokal risk of 0.89 (0.8-1.2). No treatment had been administered prior to the test.

The patient was referred for a second time in November 2013 and was treated with IM (400 mg/day) for 5 months. Thus therapeutic scheme was changed to nilotinib (600 mg/day) for 6 months with disappeared the previous reported relevant symptoms. The more recent hematological parameters were: WBC $5.3 \times 10^{9} / 1$ (50.2\% neutrophils, $48.1 \%$ lymphocytes, $0.9 \%$ monocytes and $0.8 \%$ eosinophiles). The platelet count was $118 \times 10^{9} / 1$ and the hemoglobin level was $12.4 \mathrm{~g} / \mathrm{dl}$.

\section{Results}

Pre IM treatment banding cytogenetics revealed a karyotype of $46, \mathrm{XY}, \mathrm{t}(9 ; 22)[7] / 56, \mathrm{XY},+3,+6,+7,+8,+8,+9, \mathrm{t}(9 ; 22)$, $+10,+12,+19,+\operatorname{der}(22) \mathrm{t}(9 ; 22)[5] / 57, \mathrm{XY},+3,+6,+7,+8,+8,+9$, $\mathrm{t}(9 ; 22),+10,+12,+15,-18,+19,+\operatorname{der}(22) \mathrm{t}(9 ; 22) \times 2[8]$. Post nilotinib treatment banding cytogenetics revealed a karyotype of 46,XY,t(9;22)[1]/46,XY[39] (Figure 1). Further studies were done based on molecular cytogenetics (Figure 2) and molecular genetics (Figure 3). Dual-color-FISH pre IM treatment; using a specific probe for BCR and ABL1 revealed onefusion signal on the derivative chromosomes 9 [der(9)] and another three fusion signals on the up to three Ph chromosomes (Figure 2); and chromosomes 3, 6, 7, 8, 9, 10, 12, 15, 19 and 22, were studied with WCP and/or CEP probes and did not provide any hint on cryptic translocations (data not shown). RT-PCR pre IM treatment and post nilotinib treatment confirmed the presence of the BCR-ABL1 fusion (b3a2 transcript) revealing a major $\mathrm{M}-\mathrm{BCR}$ transcript (data not shown). RQ-PCR post nilotinib treatment demonstrated a ratio of BCR-ABL/ABL1 expression of $0.07 \%$ on IS (data not shown). ASO-PCR pre IM treatment and post nilotinib treatment results showed the presence of the T315I mutation (Figure 3, (Figure 4). The final karyotype pre IM treatment and post nilotinib treatment was determined:

Pre IM treatment : 46,XY,t(9;22)(q34;q11.2)[7]/56,XY, $+3,+6,+7,+8,+8, \mathrm{t}(9 ; 22)(\mathrm{q} 34 ; \mathrm{q} 11.2),+10,+12,+19,+\operatorname{der}(22) \mathrm{t}$ $(9 ; 22)(\mathrm{q} 34 ; \mathrm{q} 11.2)[5] / 57, \mathrm{XY},+3,+6,+7,+8,+8, \mathrm{t}(9 ; 22)(\mathrm{q} 34 ;$ $\mathrm{q} 11.2),+10,+12,+15,-18,+19,+\operatorname{der}(22) \mathrm{t}(9 ; 22)(\mathrm{q} 34 ; \mathrm{q} 11.2) x 2$ [8], and post nilotinib treatment: $46, \mathrm{XY}, \mathrm{t}(9 ; 22)(\mathrm{q} 34 ; \mathrm{q} 11.2)$ $[1] / 46, \mathrm{XY}[39]$.

The abnormal cell population ( $19 \%$ blasts in peripheral blood specimen) showed the following immunophenotype, which was consistent with CML-AP (WHO recommendations): $\mathrm{CD}_{4} 5^{+}, \mathrm{CD} 34^{+}, \mathrm{CD} 10^{+}, \mathrm{CD} 19^{-}, \mathrm{CD} 20^{-}, \mathrm{CD} 22^{-}$, $\mathrm{CD}^{-}$and $\mathrm{CD}^{-}, \mathrm{HLADr}^{-}, \mathrm{CD}_{3}{ }^{+}, \mathrm{CD}_{2} 2^{+}, \mathrm{CD}^{-} 6^{-}$, $\mathrm{CD}_{117^{-}}, \mathrm{CD}_{14}^{-}, \mathrm{CD} 15^{-}, \mathrm{CD} 64^{-}$and expressed CD11c, CD38, CD235a and CD13 heterogeneously.

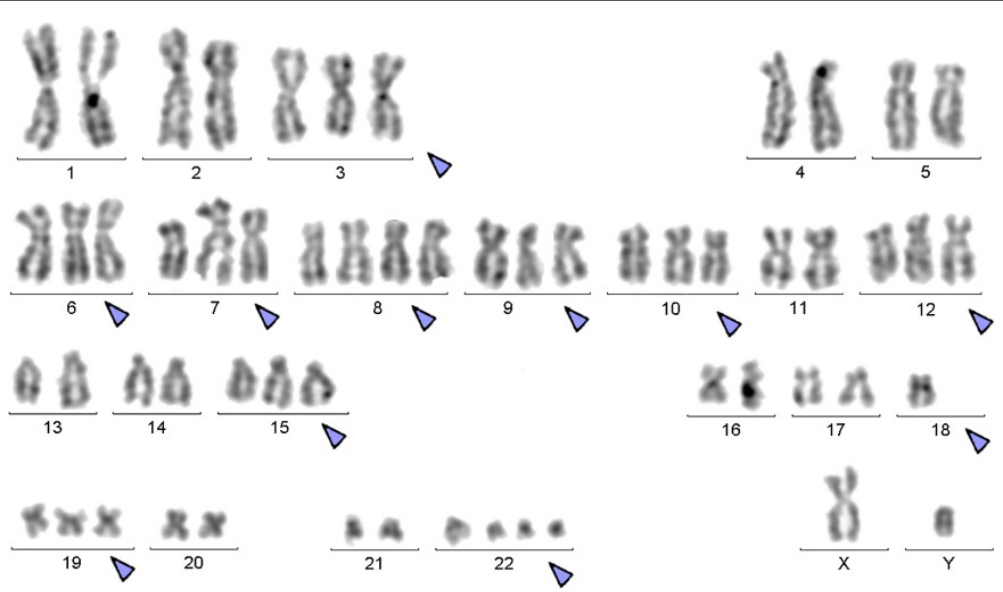

Figure 1 GTG-banding revealed a hyperdiploid karyotype. All derivative chromosomes are shown and with arrow. 


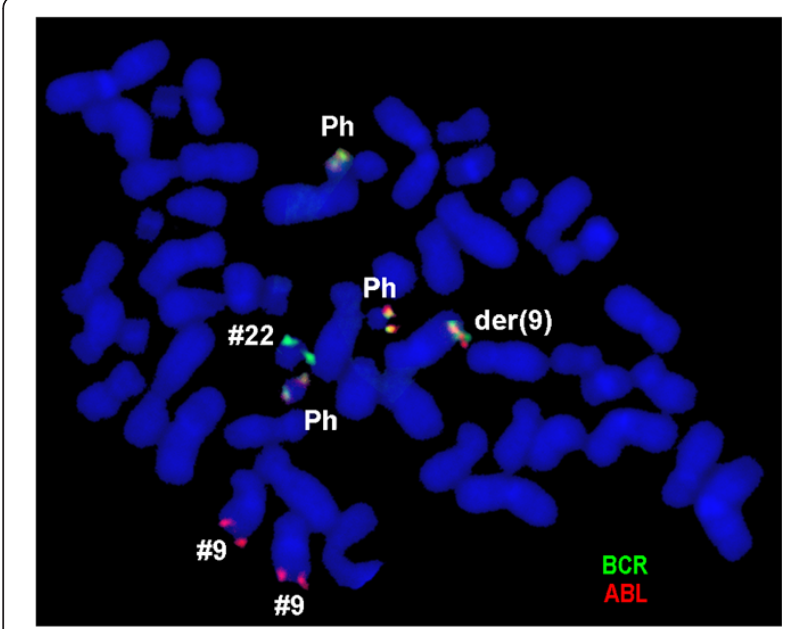

Figure 2 Partial metaphase FISH using probes for BCR (green) and $A B L$ (red) showed 4 copies of $B C R / A B L$ in this case, three copies on $\mathrm{Ph}$ chromosome and one on $\operatorname{der}(9)$. Abbreviations: \# = chromosome; der = derivative chromosome.

\section{Conclusions}

Presence of multiple copies of the $\mathrm{Ph}$ chromosome is one of several types of IM resistance mechanisms [12,13].

According to the literature, there are a few near triploid karyotypes reported in advanced phase-CML [14,15]. Stagno et al. [2] found a CML case in blast phase with hyperdiploidy and it includes $10 \%$ mutant clones with V256G mutation in BCR-ABL domain and e13a2 BCR$\mathrm{ABL}$ transcript. In this paper, we described a new $\mathrm{CML}$ case in AP with hyperdiploidy including multiple copies of $\mathrm{Ph}$ chromosome, presence of b3a2 fusion transcript and T315I mutation in the BCR-ABL KD. To the best of our knowledge, these chromosomal abnormalities, particularly hyperdiploidy associated with T315I mutation have not been previously observed in CML [16].

Approximately $10 \%$ of CML patients present in advanced phases called CML-AP or blast crisis (CML-BC), without a clinically evident CP [17]. Even with the advent of TKI therapy, the phase of disease remains an important prognostic factor in CML $[18,19]$.

Disease progression is uncommon currently in CML patients treated in CP with TKI. When it occurs, progression usually occurs early during treatment. 3.3\% of CML-CP patients progressed to CML-AP/CML-BC within the first 18 months of therapy, while $<1 \%$ progressed after 4 years of therapy [20]. The criteria for CML-AP in patients progressing on TKI therapy are still poorly defined and disease progression in CML is currently defined more practically in terms of responsiveness to TKI therapy [16].

The progression to advanced phases of disease is caused by the development of genetic lesions in addition to BCR-ABL; secondary cytogenetic changes are identified in $50-80 \%$ of CML- BC cases [21]. Also this phenomenon was described in $5-10 \%$ of patients presenting with CML$\mathrm{CP}$ and in $30 \%$ of patients developing AP features, and has overall been associated with poor prognosis [22,23]. Cytogenetic clonal evolution was subsequently considered as one of the factors defining CML-AP [24].

The most common mechanism of secondary resistance to TKI is mutation in the KD of BCR-ABL. These KD mutations develop as a result of selection pressure and correlate with progression to advanced phases and shortened survival. Thus, early identification of IM resistance is critical to allow the use of other, potentially effective therapies, such as second generation TKI and/or stem cell transplantation [25].

The mutation at amino acid 315 in the imatinibbinding site (T315I mutation) confers resistance to imatinib, dasatnib, and nilotinib by preventing access of these drugs to the ATP-binding pocket [26-28]. Hughes et al. [29] found six cases (5 IM resistant and one IM intolerant, i.e. six in total) with T315I mutation and two of the six cases did not showed responses to Nilotinib but couldn't sustain. In addition, Jabbour et al. [30] found

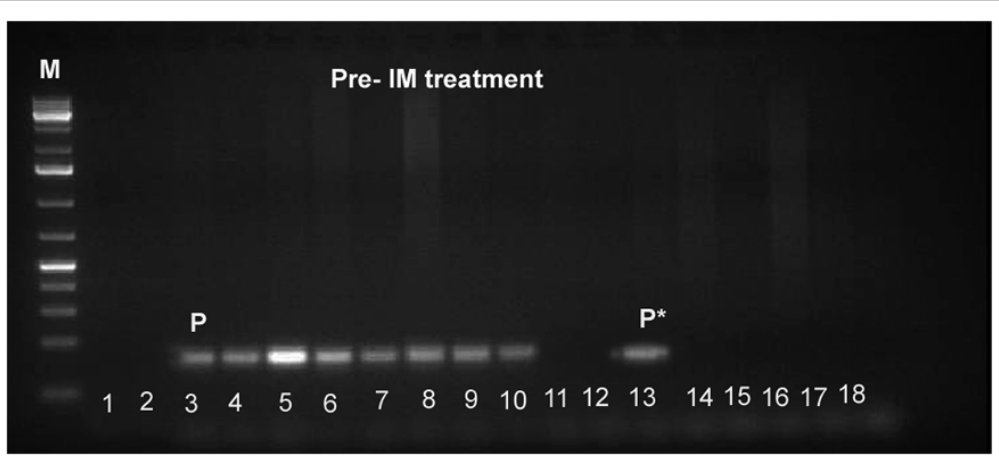

Figure 3 ASO-PCR monitoring and corresponding sensitivity test for detected T315I mutation pre-IM treatment. ASO-PCR products on genomic DNA obtained from healthy donor and our patient. Line M, 1 kb DNA ladder; lines 1,2 blank; lines 3-10 patients ASO-PCR T315I wild-type primers; P, our patient; lines 11-18 ASO-PCR T315I mutated primers; and P*, our patient with T315I mutation. 


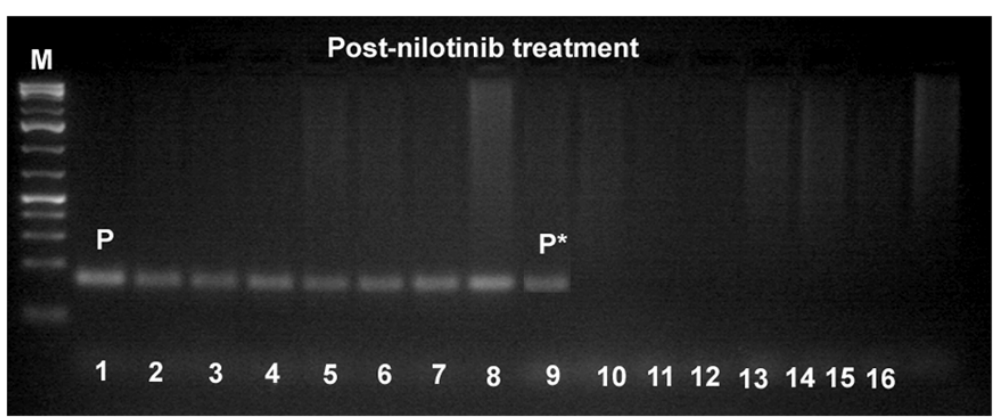

Figure 4 ASO-PCR monitoring and corresponding sensitivity test for detected T315I mutation Post-nilotinib treatment. Line M, $1 \mathrm{~kb}$ DNA ladder; lines 1-8 patients ASO-PCR T315I wild-type primers; P, our patient; lines 9-16 ASO-PCR T315I mutated primers; and P*, our patient with T315I mutation.

one case of CML-AP with T315I mutation and complete cytogenetic response to Nilotinib. Also, Jabbour et al. [31] found of CML-AP with T315I mutation and Nilotinib resistance. However, T315I mutation also was observed in 10 cases post IM failure (4 CP, $5 \mathrm{AP}$, and 1 blast phase) and 2 cases post Nilotinib treatment in the study by Cortes et al. [32]. Cea et al. [33] also found T315I mutation post IM, post Nilotinib and during Dasatinib treatment in e19a2 BCR/ABL1 CML. Our patient achieved a major cytogenetic response and major molecular response after 6 months of nilotinib treatment with T315I mutation remaining.

The mechanisms by which these mutations develop are poorly understood. KD mutations can be present at low-levels in CML cases prior to exposure to TKI therapy. However, these small mutant clones do not necessarily confer an adverse prognosis and resistant clones may not expand even under the selection pressure of TKI therapy $[34,35]$. The KD mutations usually occur within the first 2 years of TKI therapy and are much more common in CML-AP and CML-BC than CML-CP [7].

In conclusion, we described a new CML case in AP pre IM treatment with hyperdiploidy including multiple copies of Ph chromosome, and presence of T315I mutation in the BCR-ABL KD. The patient demonstrated a good response to nilotinib treatment after imatinib course failure. Interestingly hyperdiploidy clone disappeared while the T315I mutation remained.

\section{Materials and methods Chromosome analysis}

Chromosome analysis applying GTG-banding according to standard procedures [36] was performed pre IM treatment and post nilotinib treatment. 20 metaphase cells derived from unstimulated bone marrow culture were analyzed. Karyotypes were described according to the International System for Human Cytogenetic Nomenclature (ISCN 2009).

\section{Molecular cytogenetics}

Fluorescence in situ hybridization (FISH) using the LSI $\mathrm{BCR} / \mathrm{ABL}$ dual color dual fusion translocation probe (Abbott Molecular/Vysis, Des Plaines, IL, USA) and/or chromosome enumeration probes (CEP) for chromosomes 6, 7, 8 and/or 12 (Abbott Molecular/Vysis, Des Plaines, IL, USA) were applied together with whole chromosome painting (WCP) probes for chromosomes 3, 9, 10, 15, 19 and/or 22 (MetaSystems, Altlussheim, Germany) according to manufacturer's instructions [36]. A minimum of 10 metaphase spreads was analyzed, using a fluorescence microscope (AxioImager.Z1 mot, Carl Zeiss Ltd., Hertfordshir, UK) equipped with appropriate filter sets to discriminate between a maximum of five fluorochromes plus the counterstain DAPI (4',6- diamino-2-phenylindole). Image capture and processing were performed using an ISIS imaging system (MetaSystems, Altlussheim, Germany).

Reverse transcriptase-polymerase chain reaction (RT-PCR) and quantitative reverse transcription polymerase chain reaction (RQ-PCR) for $B C R / A B L 1$ fusion transcripts Total RNA extracted from peripheral blood sample using the InviTrap RNA kit (Invitek, Berlin, Germany) according to the manufacturer's recommendations. cDNA was prepared from $5 \mu \mathrm{g}$ of total RNA with the Genequality BCRABL1 kit (AB Analitica, Padova, Italy) and BCR-ABL1 fusion transcript was performed according to the manufacturer's instructions (AB Analitica, Padova, Italy). RQPCR analyses were performed in StepOne Real-Time PCR (Applied Biosystems, Foster City, California, USA) using one-step qRT-PCR BCR-ABL1 kit (MolecularMD, Portland, USA) according to the manufacturer's recommendations.

\section{Allele-specific oligonucleotide (ASO)-PCR}

Mutated or wild-type sequences were amplified in a noncompetitive PCR reaction performed on genomic DNA and PCR conditions using allele-specific and 
reverse primers for the Thr315Ile mutation as described previously. The length of the PCR product using $1 \mathrm{~Kb}$ DNA ladder (Fermentas, Lithuania, Vilnius) was (158 bp). The sensitivity of this assay was determined for each mutation by amplification of 10 -fold limited dilutions of $100 \mathrm{ng}$ patient's DNA at time of resistance in $100 \mathrm{ng}$ healthy control DNA [37].

\section{Flow cytometricimmunophenotype}

Flow cytometry of leukemic blasts was performed using a general panel of fluorescent antibodies against the following antigens typical for different cell lineages and cell types: CD1a, CD2, CD3, CD4, CD5, CD8, CD10, CD11b, CD11c, CD13, CD14, CD15, CD16, CD19, CD20, CD22, CD23, CD32, CD33, CD34, CD38, CD41a, CD45, CD56, CD57, CD64, CD103, CD117, CD123, CD209, CD235a and CD243; in addition antibodies against Kappa and Lambda light Chains, sIgD, sIgM, and HLADr were applied (BD Biosciences). Four-color immunophenotyping on peripheral blood specimen was performed. Samples were stained and analyzed on a BD FACSCalibur ${ }^{\text {Tx }}$ flow cytometer according to BD Biosciences manuals and products insert sheets. Autofluorescence, viability, and isotype controls were included. Flow cytometric data acquisition and analysis were conducted by BD Cellquest ${ }^{\mathrm{tm}}$ Pro software.

\section{Consent}

Written informed consent was obtained from the patient for publication of this Case Report. A copy of the written consent is available for review by the Editor-in-Chief of this journal.

\section{Competing interests}

The authors declare that they have no competing interests.

\section{Authors' contributions}

AW and FM provided the case and/or did primary cytogenetic and main part of the FISH-tests; AA did the flow cytometry analysis; TL and MAKO did detailed FISH studies. WA supervised the cytogenetic analysis as Director of the MBBD. AW and TL drafted the paper and all authors read and approved the final manuscript.

\section{Acknowledgements}

We thank Prof. I. Othman, the Director General of Atomic Energy Commission of SYRIA (AECS) and Dr. N. Mirali, Head of Molecular Biology and Biotechnology Department for their support. This work was supported by the AECS, in parts by the DAAD.

\section{Author details \\ 'Human Genetics Division, Department of Molecular Biology and Biotechnology, Atomic Energy Commission, P.O. Box 6091, Damascus, Syria. ${ }^{2}$ Mammalians Biology Division, Department of Molecular Biology and Biotechnology, Atomic Energy Commission, Damascus, Syria. ${ }^{3}$ Institute of Human Genetics, Jena University Hospital, Jena, Germany.}

Received: 28 September 2014 Accepted: 11 November 2014 Published online: 29 November 2014

\section{References}

1. Johansson B, Fioretos T, Mitelman F: Cytogenetic and molecular genetic evolution of chronic myeloid leukemia. Acta Haematol 2002, 107:76-94.
2. Stagno F, Vigneri $P$, Consoli ML, Cupri A, Stella S, Tambè L, Massimino M, Manzella L, Di Raimondo F: Hyperdiploidy associated with a high BCR-ABL transcript level may identify patients at risk of progression in chronic myeloid leukemia. Acta Haematol 2012, 127:7-9.

3. Belurkar S, Manohar C, Kurien A: Chronic myeloid leukemia with hyperdiploidy: a case report with review of literature. Indian J Med SCi 2013, 67:188-192.

4. Calabretta B, Perrotti D: The biology of CML blast crisis. Blood 2004, 103:11.

5. Roland B, Blahey Walter B: A case of near triploidy in chronic myelogenousleukemia. Cancer Genet Cytogenet 2000, 121:96-98.

6. Fabarius A, Leitner A, Hochhaus A, Müller MC, Hanfstein B, Haferlach C, Göhring G, Schlegelberger B, Jotterand M, Reiter A, Jung-Munkwitz S, Proetel U, Schwaab J, Hofmann WK, Schubert J, Einsele H, Ho AD, Falge C, Kanz L, Neubauer A, Kneba M, Stegelmann F, Pfreundschuh M, Waller CF, Spiekermann K, Baerlocher GM, Lauseker M, Pfirrmann M, Hasford J, Saussele S, Hehlmann R, SchweizerischeArbeitsgemeinschaftfürKlinischeKrebsforschung (SAKK) and the German CML Study Group: Impact of additional cytogenetic aberrations at diagnosis on prognosis of CML: long-term observation of 1151 patients from the randomized CML Study IV. Blood 2011, 118:6760-6768.

7. Druker BJ, Guilhot F, O'Brien SG, Gathmann I, Kantarijan H, Gattermann N, Deininger MW, Silver RT, Goldman JM, Stone RM, Cervantes F, Hochhaus A, Powell BL, Gabrilove JL, Rousselot P, Reiffers J, Cornelissen JJ, Hughes T, Agis H, Fischer T, Verhoef G, Shepherd J, Saglio G, Gratwohl A, Nielsen JL, Radich JP, Simonsson B, Taylor K, Baccarani M, So C, Letvak L, Larson RA, IRIS Investigators: Five-year follow-up of patients receiving imatinib for chronic myeloid leukemia. NEngl J Med 2006, 355:2408-2417.

8. Weisberg E, Manley P, Mestan J, Cowan-Jacob S, Ray A, Griffin JD: AMN107 (nilotinib): a novel and selective inhibitor of BCR-ABL. Br J Cancer 2006, 94:765-1769.

9. Tojo A, Usuki K, Urabe A, Maeda Y, Kobayashi Y, Jinnai I, Ohyashiki K, Nishimura M, Kawaguchi T, Tanaka H, Miyamura K, Miyazaki Y, Hughes T, Branford S, Okamoto S, Ishikawa J, Okada M, Usui N, Tanii H, Amagasaki T, Natori $\mathrm{H}$, Naoe T: A phase I/II study of nilotinib in Japanese patients with imatinib-resistant or- intolerant $\mathrm{Ph}+\mathrm{CML}$ or relapsed/refractory $\mathrm{Ph}+$ ALL. Int J Hematol 2009, 89:679-688.

10. Cang S, Liu D: P-loop mutations and novel therapeutic approaches for imatinib failures in chronic myeloid leukemia. J Hematol Oncol 2008, 1:15.

11. Branford S, Melo JV, Hughes TP: Selecting optimal second-line tyrosine kinase inhibitor therapy for chronic myeloid leukemia patients after imatinib failure: does the BCR-ABL mutation status really matter? Blood 2009, 114:5426-5435.

12. Otero L, Ornellas MH, Dobbin J, de Souza FT: Double Philadelphiachromosome: a resistance factor on the imatinibmesylate therapy for chronic myeloid leukemia. Int I Lab Hematol 2008, 30:346-348.

13. Ossard- Receveur A, Bernheim A, Clausse B, Danglot G, Fauvet D, Leon B, Lozach F: Duplication of the Ph-chromosome as a possible mechanism of resistance to imatinibmesylate in patients with chronic myelogenous leukemia. Cancer Genet Cytogenet 2005, 163:189-190.

14. Amiel A, Yukla M, Gaber E, Leopold L, Josef G, Fejgin M, Lishner M: Random aneuploidy in CML patients at diagnosis and under imatinibtreatment. Cancer Genet Cytogenet 2006, 168:120-123.

15. Sheth FJ, Sheth JJ, Desai C: Case of near triploidy with $\mathrm{i}(17)(\mathrm{q} 10)$ in blast crisis CML. Cancer Genet Cytogenet 2006, 164:177-178.

16. Baccarani M, Saglio G, Goldman J, Hochhaus A, Simonsson B, Appelbaum F, Apperley J, Cervantes F, Cortes J, Deininger M, Gratwohl A, Guilhot F, Horowitz M, Hughes T, Kantarjian H, Larson R, Niederwieser D, Silver R, Hehlmann R, European LeukemiaNet: Evolving concepts in the management of chronic myeloid leukemia: recommendations from an expert panel on behalf of the European Leukemia- Net. Blood 2006, 108:1809-1820,

17. Quintas-Cardama A, Cortes JE: Chronic myeloid leukemia: diagnosis and treatment. Mayo Clin Proc 2006, 81:973-988.

18. Jabbour E, Kantarjian H, Jones D, Talpaz M, Bekele N, O'Brien S, Zhou X, Luthra R, Garcia-Manero G, Giles F, Rios MB, Verstovsek S, Cortes J: Frequency and clinical significance of BCR-ABL mutations in patients with chronic myeloid leukemia treated with imatinibmesylate. Leukemia 2006, 20:1767-1773.

19. Mitchell B, Deininger M: Techniques for risk stratification of newly diagnosed patients with chronic myeloid leukemia. Leuk Lymphoma 2011, 52:4-11. 
20. O'Brien SG, Guilhot F, Larson RA, Gathmann I, Baccarani M, Cervantes F, Cornelissen JJ, Fischer T, Hochhaus A, Hughes T, Lechner K, Nielsen JL, Rousselot P, Reiffers J, Saglio G, Shepherd J, Simonsson B, Gratwohl A, Goldman JM, Kantarjian H, Taylor K, Verhoef G, Bolton AE, Capdeville R, Druker BJ, IRIS Investigators: Imatinib compared with interferon and lowdose cytarabine for newly diagnosed chronic-phase chronic myeloid leukemia. N Engl J Med 2003, 348:994-1004

21. Feng J, Le Beau MM, Larson RA, Rowley JD, Vardiman JW: The relationship between secondary chromosomal abnormalities and blast transformation in chronic myelogenousleukemia. Leukemia 1995, 9:628-633.

22. Swolin B, Weinfeld A, Westin J, Waldenström J, Magnusson B: Karyotypic evolution in $\mathrm{Ph}$-positive chronic myeloid leukemia in relation to management and disease progression. Cancer Genet Cytogenet 1985 18:65-79.

23. Sokal JE, Gomez GA, Baccarani M, Tura S, Clarkson BD, Cervantes F, Rozman C, Carbonell F, Anger B, Heimpel H: Prognostic significance of additional cytogenetic abnormalities at diagnosis of Philadelphia chromosomepositive chronic granulocytic leukemia. Blood 1988, 72:294-298.

24. Kantarjian HM, Dixon D, Keating MJ, Talpaz M, Walters RS, McCredie KB, Freireich EJ: Characteristics of accelerated disease in chronic myelogenousleukemia. Cancer 1988, 61:1441-1446.

25. Soverini S, Martinelli G, Rosti G, Bassi S, Amabile M, Poerio A, Giannini B, Trabacchi E, Castagnetti F, Testoni N, Luatti S, de Vivo A, Cilloni D, Izzo B, Fava M, Abruzzese E, Alberti D, Pane F, Saglio G, Baccarani M: ABL mutations in late chronic phase chronic myeloid leukemia patients with upfront cytogenetic resistance to imatinib are associated with a greater likelihood of progression to blast crisis and shorter survival: a study by the GIMEMA Working Party on Chronic Myeloid Leukemia. J Clin Oncol 2005, 23:4100-4109.

26. Hasserjian RP: Chronic myelogenous leukemia in the age of imatinib: assessing response, acceleration, and blast phase. J Hematopathol 2011, 4:81-92.

27. Apperley JF, Cortes JE, Kim DW, Roy L, Roboz GJ, Rosti G, Bullorsky EO, Abruzzese E, Hochhaus A, Heim D, de Souza CA, Larson RA, Lipton JH, Khoury HJ, Kim HJ, Sillaber C, Hughes TP, Erben P, Van Tornout J, Stone RM: Dasatinib in the treatment of chronic myeloid leukemia in accelerated phase after imatinib failure: the START A trial. J Clin Oncol 2009, 27:3472-3479.

28. O'Hare T, Walters DK, Stoffregen EP, Jia T, Manley PW, Mestan J, CowanJacob SW, Lee FY, Heinrich MC, Deininger MW, Druker BJ: In vitro activity of Bcr-Abl inhibitors AMN107 and BMS-354825 against clinically relevant imatinibresistantAbl kinase domain mutants. Cancer Res 2005, 65:4500-4505.

29. Hughes T, Saglio G, Branford S, Soverini S, Kim DW, Müller MC, Martinelli G, Cortes J, Beppu L, Gottardi E, Kim D, Erben P, Shou Y, Haque A, Gallagher N, Radich J, Hochhaus A: Impact of baseline BCR-ABL mutations on response to nilotinib in patients with chronic myeloid leukemia in chronic phase. J Clin Oncol 2009, 27:4204-4210.

30. Jabbour E, Kantarjian H, Jones D, Breeden M, Garcia-Manero G, O'Brien S, Ravandi F, Borthakur G, Cortes J: Characteristics and outcomes of patients with chronic myeloid leukemia and T315I mutation following failure of imatinibmesylatetherapy. Blood 2008, 112:53-55.

31. Jabbour E, Cortés JE, Kantarjian H: Second-line therapy and beyond resistance for the treatment of patients with chronic myeloid leukemia post imatinibfailure. Clin Lymphoma Myeloma 2009, 9:S272-S279.

32. Cortes J, Jabbour E, Kantarjian H, Yin CC, Shan J, O'Brien S, Garcia-Manero G, Giles F, Breeden M, Reeves N, Wierda WG, Jones D: Dynamics of BCR-ABL kinase domain mutations in chronic myeloid leukemia after sequential treatment with multiple tyrosine kinase inhibitors. Blood 2007, 110:4005-4011.

33. Cea M, Cirmena G, Garuti A, Rocco I, Palermo C, Cagnetta A, Moran E, Colombo N, Grasso R, Fugazza G, Gobbi M, Nencioni A, Ballestrero A, Patrone F: A T315I mutation in e19a2 BCR/ABL1 chronic myeloid leukemia responding to dasatinib. Leuk Res 2010, 34:e240-e242.

34. O'Hare T, Eide CA, Deininger MW: Bcr-Abl kinase domain mutations, drug resistance, and the road to a cure for chronic myeloid leukemia. Blood 2007, 110:2242-2249.

35. Willis SG, Lange T, Demehri S, Otto S, Crossman L, Niederwieser D, Stoffregen EP, McWeeney S, Kovacs I, Park B, Druker BJ, Deininger MW: High-sensitivity detection of BCR-ABL kinase domain mutations in imatinib-naive patients: correlation with clonal cytogenetic evolution but not response to therapy. Blood 2005, 106:2128-2137.
36. AL-achkar W, Wafa A, Nweder MS: A complex translocation $t(5 ; 9 ; 22)$ in Philadelphia cells involving the short arm of chromosome 5 in a case of chronic myelogenousleukemia. J Exp Clin Cancer Res 2007, 26:411-415.

37. Roche-Lestienne C, Soenen-Cornu V, Grardel-Duflos N, Laï JL, Philippe N, Facon T, Fenaux P, Preudhomme C: Several types of mutations of the Abl gene can be found in chronic myeloid leukemia patients resistant to STI571, and they can pre-exist to the onset of treatment. Blood 2002, 100:1014-1018.

\section{Submit your next manuscript to BioMed Central and take full advantage of:}

- Convenient online submission

- Thorough peer review

- No space constraints or color figure charges

- Immediate publication on acceptance

- Inclusion in PubMed, CAS, Scopus and Google Scholar

- Research which is freely available for redistribution

Submit your manuscript at www.biomedcentral.com/submit
C) BioMed Central 